\title{
Cross-correlation between photons and phonons in quadratically coupled optomechanical systems
}

\author{
Xun-Wei Xu, ${ }^{1, *}$ Hai-Quan Shi, ${ }^{2,1}$ Ai-Xi Chen, ${ }^{3,1, \dagger}$ and Yu-xi Liu ${ }^{4,5}$ \\ ${ }^{1}$ Department of Applied Physics, East China Jiaotong University, Nanchang, 330013, China \\ ${ }^{2}$ School of Materials Science and Engineering, Nanchang University, Nanchang 330031, China \\ ${ }^{3}$ Department of Physics, Zhejiang Sci-Tech University, Hangzhou, 310018, China \\ ${ }^{4}$ Institute of Microelectronics, Tsinghua University, Beijing 100084, China \\ ${ }^{5}$ Beijing National Research Center for Information Science and Technology (BNRist), Beijing 100084, China
}

(Dated: July 16, 2018)

\begin{abstract}
We study photon, phonon statistics and the cross-correlation between photons and phonons in a quadratically coupled optomechanical system. Photon blockade, phonon blockade, and strong anticorrelation between photons and phonons can be observed in the same parameter regime with the effective nonlinear coupling between the optical and mechanical modes, enhanced by a strong optical driving field. Interestingly, an optimal value of the effective nonlinear coupling strength for the photon blockade is not within the strong nonlinear coupling regime. This abnormal phenomenon results from the destructive interference between different paths for twophoton excitation in the optical mode with a moderate effective nonlinear coupling strength. Furthermore, we show that phonon (photon) pairs or correlated photons and phonons can be generated in the strong nonlinear coupling regime with a proper detuning between the weak mechanical driving field and mechanical mode. Our results open up a way to generate anticorrelated and correlated photons and phonons, which may have important applications in quantum information processing.
\end{abstract}

\section{INTRODUCTION}

To fulfill the needs of quantum information processing [1, 2], the development of single photon sources [3-5] has become a research focus in quantum physics. A perfect single photon source can emit a single photon in one time so that the emitted photons show strong antibunching effect. Photon blockade [6] that the excitation of the first photon blocks the excitation of the second photon can be used to realize perfect single photon source. Photon blockade has been observed in many different systems, such as cavity quantum electrodynamics systems [7-9], a quantum dot in a photonic crystal $[10,11]$, and circuit quantum electrodynamics system [1214].

As the counterpart of photons, phonons are the elementary excitations in mechanical systems. With the development of micro- and nano-technology, such as improving the quality factor of the mechanical oscillators and enhancing the coupling strengths of mechanical oscillators to other quantum systems [15, 16], the appearance of phonon lasers [17-19] and theoretical proposals on generating single phonons [2028], phonons gradually become a new candidate for quantum information processing. Moreover, the measurement of the correlations of the phonons can be realized by converting the mechanical signals into optical signals through auxiliary optomechanical couplings [23-25]. In a recent experiment [24], the phonon correlation in an optomechanical system has been measured by detecting the correlations of the emitted photons from the optical cavity.

As the complexity of the tasks, entrusted to the quantum information processors, becomes higher and higher, hybrid optical and mechanical systems become more and more important

\footnotetext{
*Electronic address: davidxu0816@163.com

†Electronic address: aixichen@ecjtu.edu.cn
}

$[29,30]$. One useful approach to improve the overall coordination is to design a hybrid device which can generate not only single photons and single phonons, but also correlated single photons and single phonons. The coupling between optical and mechanical systems based on two main ways: one is induced indirectly by muti-level atoms, such as artificial atoms based superconducting quantum circuits $[30,31]$ and nitrogen-vacancy centers $[32,33]$; the other one is induced by parameters coupling, such as optomechanical coupling [34].

Optomechanical system, that a cavity mode is coupled to a mechanical mode via radiation pressure or optical gradient forces, provides us an appropriate platform to manipulate both photons and phonons simultaneously (for reviews, see Refs. [35-40]). It has been shown that photon blockade can be realized in the optomechanical system with different structures, such as strongly coupled optomechanical systems at single-photon level [41-47], multimode optomechanical systems [48-51], squeezed optomechanical systems [52, 53], quadratically coupled optomechanical system driven by a strong optical field [54]. Recently, strong phonon antibunching was proposed in a quadratically coupled optomechanical system [55-57] and this provides another possible way to generate single phonons in the optomechanical system. However, the realization of both photon blockade and phonon blockade in one optomechanical system with the same parameters has not been considered yet. Moreover, optomechanical system provides us an ideal platform to investigate the crosscorrelation between photons and phonons [58], which has important applications in quantum information processing.

In this paper, we study photon statistics, phonon statistics and the cross-correlation between photons and phonons in a quadratically coupled optomechanical system. The effective nonlinear coupling between the optical and mechanical modes in a quadratically coupled optomechanical system can be enhanced by a strong driving optical field as shown in Refs. [54, 56, 57, 59]. Different from the previous studies $[54,56]$, we find that there is an optimal value of the effec- 
tive nonlinear coupling strength for photon blockade before reaching the strong nonlinear coupling regime, and both photon and phonon blockades can be observed in a quadratically coupled optomechanical system with the same parameters. Moreover, we also study the cross-correlation between photons and phonons, and show that both anticorrelated and correlated phonons and photons can be generated in the quadratically coupled optomechanical system.

The paper is organized as follows. In Sec. II, we show the theoretical model of the quadratically coupled optomechanical system. In Sec. III, we study phonon blockade, photon blockade, anti-correlation between phonons and photons, and the effects of the parameters on the statistical properties of the system are discussed. In Sec. IV, we explain how the strong photon blockade appears with a weak nonlinear coupling strength. In Sec. $\mathrm{V}$, we show that phonon (photon) pairs and correlated photons and phonons can be generated in the strong nonlinear coupling regime with a proper detuning between the weak mechanical driving field and mechanical mode. Finally, we summarize the results in Sec. VI.

\section{THEORETICAL MODEL}

We study an optomechanical system in which a mechanical mode is quadratically coupled to an optical mode. Such system can be found in the optomechanical crystals [60], Fabry-Perot cavities with membrane-in-the-middle [61-64], and other optomechanical systems [65-68]. We assume that the optical mode is driven by an external field with the strength $\Omega$ and frequency $\omega_{L}$, and the mechanical mode is driven by a mechanical pump of strength $2 \sqrt{2} \varepsilon \cos \left(\omega_{d} t\right)$ (amplitude $\varepsilon$, frequency $\omega_{d}$ ). In the rotating reference frame with optical frequency $\omega_{L}$, the system can be described by a Hamiltonian $(\hbar=1)$

$$
\begin{aligned}
H= & \Delta_{c} A^{\dagger} A+\frac{1}{2} \omega_{m}\left(Q^{2}+P^{2}\right)+2 g A^{\dagger} A Q^{2} \\
& +\left(\Omega A^{\dagger}+\text { H.c. }\right)+2 \sqrt{2} Q \varepsilon \cos \left(\omega_{d} t\right)
\end{aligned}
$$

where $\Delta_{c}=\omega_{c}-\omega_{L}$ is the detuning of the strong optical driving field from the optical mode with frequency $\omega_{c} ; A$ and $A^{\dagger}$ are the annihilation and creation operators of the optical mode, $Q$ and $P$ are the dimensionless displacement and momentum operators of the mechanical mode with frequency $\omega_{m}$, and $g>0$ is the quadratic optomechanical coupling strength between the optical and mechanical modes. The damping rates of the optical mode and mechanical mode are $\gamma_{c}$ and $\gamma_{m}$, respectively. We assume that the strength of the optical driving field is strong, i.e., $\Omega \gg \gamma_{c}$, while the strength of the mechanical driving field is much weaker than the damping rate of the optical mode, i.e., $\varepsilon \ll \gamma_{c}$.

Based on the Hamiltonian in Eq. (1), a tunable second-order nonlinear coupling between the optical and mechanical modes can be induced by the strong optical driving field. The quantum Langevin equations (QLEs) for the operators are given by

$$
\frac{d A}{d t}=-\left(i \Delta_{c}+\frac{\gamma_{c}}{2}\right) A-i 2 g A Q^{2}-i \Omega+\sqrt{\gamma_{c}} A_{\mathrm{in}}
$$

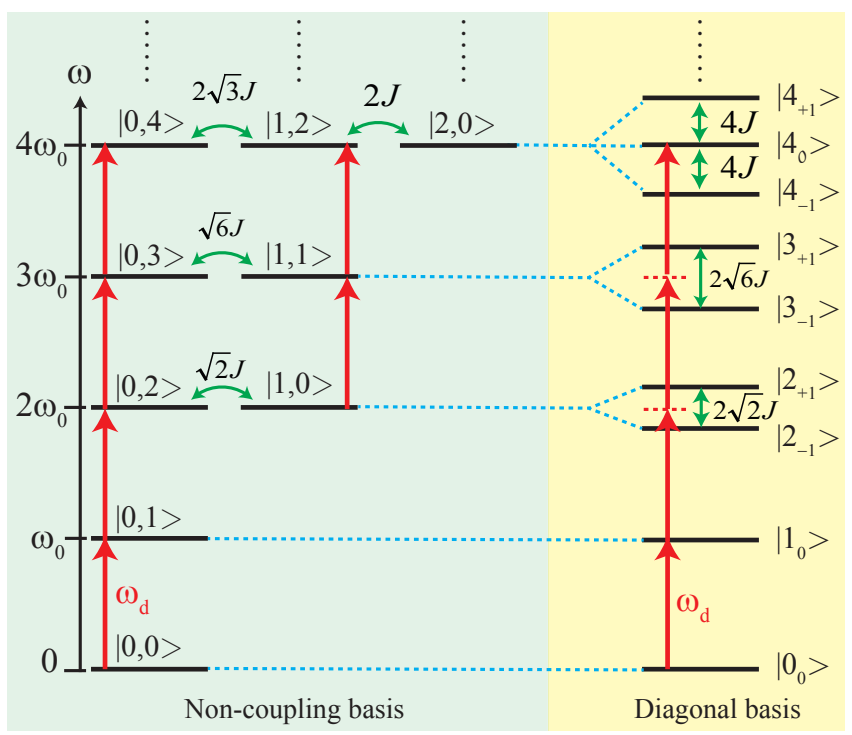

FIG. 1: (Color online) The schematic energy spectrum of the linearized quadratically coupled optomechanical system [see the Hamiltonian in Eq. (8)] given in the non-coupling basis (left) and in the diagonal basis (right).

$$
\begin{gathered}
\frac{d Q}{d t}=\omega_{m} P, \\
\frac{d P}{d t}=-\omega_{m} Q-4 g A^{\dagger} A Q-\frac{\gamma_{m}}{2} P-2 \sqrt{2} \varepsilon \cos \left(\omega_{d} t\right)+\xi,
\end{gathered}
$$

where $A_{\text {in }}$ and $\xi$ are the noise operators with zero mean values. The steady state mean values $\alpha, Q_{s}$ and $P_{s}$ of operators $A, Q$ and $P$ can be obtained by taking the quantum average of the QLEs and setting the time derivatives to zeros. Without considering the weak mechanical driving field, i.e., $\varepsilon=0$, the steady state mean values $\alpha, Q_{s}$ and $P_{s}$ for $g>0$ are shown as

$$
\begin{gathered}
\alpha=\frac{-i 2 \Omega}{\gamma_{c}+i 2 \Delta_{c}}, \\
Q_{s}=P_{s}=0 .
\end{gathered}
$$

We expand the operators as the sum of their steady state mean values and quantum fluctuations: $A \rightarrow \alpha+a, Q \rightarrow Q_{s}+q$ and $P \rightarrow P_{s}+p$, where $a, q$ and $p$ are the quantum flucturation operators, then the effective Hamiltonian $H^{\prime}$ for the quantum flucturation operators reads

$$
\begin{aligned}
H^{\prime}= & \Delta_{c} a^{\dagger} a+\omega_{m} b^{\dagger} b+g\left(|\alpha|^{2}+a^{\dagger} a\right)\left(b^{\dagger}+b\right)^{2} \\
& +g\left(\alpha a^{\dagger}+\alpha^{*} a\right)\left(b^{\dagger}+b\right)^{2} \\
& +2 \varepsilon \cos \left(\omega_{d} t\right)\left(b^{\dagger}+b\right),
\end{aligned}
$$

where $q \equiv\left(b^{\dagger}+b\right) / \sqrt{2}, p \equiv i\left(b^{\dagger}-b\right) / \sqrt{2}$. For a strong optical driving field, we assume that the steady-state mean value $\alpha$ is much larger than the quantum flucturation operators $a$, such as $|\alpha|^{2} \gg\left\langle a^{\dagger} a\right\rangle$, then the term $g a^{\dagger} a\left(b^{\dagger}+b\right)^{2}$ 
in the above equation can be neglected. In the rotating reference frame with respect to the unitary operator $R(t)=$ $\exp \left(i 2 \omega_{d} a^{\dagger} a t+i \omega_{d} b^{\dagger} b t\right)$, under the rotating-wave approximation by neglecting the terms oscillating with high frequencies in Eq. (7), e.g. $2 \omega_{d}$ and $4 \omega_{d}$, a simplified effective Hamiltonian is obtained as

$$
H_{\mathrm{eff}}=\Delta a^{\dagger} a+\Delta_{m} b^{\dagger} b+J a^{\dagger} b^{2}+J^{*} a b^{\dagger 2}+\left(\varepsilon b^{\dagger}+\text { H.c. }\right),
$$

where the detunings $\Delta=\Delta_{c}-2 \omega_{d}$ and $\Delta_{m}=\omega_{m}+2 g|\alpha|^{2}-$ $\omega_{d}$ satisfy the condition $\left\{|\Delta|,\left|\Delta_{m}\right|\right\} \ll \omega_{m} ; J=g \alpha$ is the effective second-order nonlinear coupling strength between the optical and mechanical modes, and can be controlled by tuning the strength of the strong optical driving field. Without loss of generality $J$ is assumed to be real.

The energy spectrum of the Hamiltonian for the linearized quadratically coupled optomechanical system in Eq. (8) is shown in Fig. 1, where $\omega_{0} \equiv \omega_{m}+2 g|\alpha|^{2}=\Delta_{c} / 2$. In the non-coupling basis (left), $|n, m\rangle$ represents the Fock state with $n$ photons in optical mode and $m$ phonons in the mechanical mode. In the diagonal basis (right), we have $\left|0_{0}\right\rangle \equiv|0,0\rangle,\left|1_{0}\right\rangle \equiv|0,1\rangle,\left|2_{ \pm 1}\right\rangle \equiv(|1,0\rangle \pm|0,2\rangle) / \sqrt{2}$, $\left|3_{ \pm 1}\right\rangle \equiv(|1,1\rangle \pm|0,3\rangle) / \sqrt{2},\left|4_{0}\right\rangle \equiv(-\sqrt{3}|2,0\rangle+|0,4\rangle) / 2$ and $\left|4_{ \pm 1}\right\rangle \equiv(|2,0\rangle \pm 2|1,2\rangle+\sqrt{3}|0,4\rangle) /(2 \sqrt{2})$. To have $J \sim \gamma_{c}$ in the weak quadratically coupling regime $g \ll \gamma_{c}$, the optical mode has to be strongly driven with $|\alpha| \gg 1$. In this case, we have the frequency shift $\omega^{\prime} \equiv 2 g|\alpha|^{2} \approx$ $2|J \alpha| \gg \gamma_{c}$ and the effective frequency of the mechanical mode $\omega_{0}=\omega_{m}+\omega^{\prime}$ may be much higher than the bare frequency of the mechanical mode $\omega_{m}$.

To quantify the statistics of the phonons and photons in the system, we consider the second-order correlation functions in the steady state $(t \rightarrow \infty)$ defined by

$$
\begin{aligned}
& g_{a a}^{(2)}(\tau) \equiv \frac{\left\langle a^{\dagger}(t) a^{\dagger}(t+\tau) a(t+\tau) a(t)\right\rangle}{n_{a}(t)^{2}}, \\
& g_{b b}^{(2)}(\tau) \equiv \frac{\left\langle b^{\dagger}(t) b^{\dagger}(t+\tau) b(t+\tau) b(t)\right\rangle}{n_{b}(t)^{2}}, \\
& g_{a b}^{(2)}(\tau) \equiv\left\{\begin{array}{ll}
\frac{\left\langle a^{\dagger}(t) b^{\dagger}(t+\tau) b(t+\tau) a(t)\right\rangle}{n_{a}(t) n_{b}(t)} & \tau \geq 0 \\
\frac{\left\langle b^{\dagger}(t) a^{\dagger}(t-\tau) a(t-\tau) b(t)\right\rangle}{n_{a}(t) n_{b}(t)} & \tau<0
\end{array},\right.
\end{aligned}
$$

where $n_{a}(t) \equiv\left\langle a^{\dagger}(t) a(t)\right\rangle$ and $n_{b}(t) \equiv\left\langle b^{\dagger}(t) b(t)\right\rangle$ are the mean photon and phonon numbers. The dynamic behavior of the total open system is described by the master equation for the density matrix $\rho$ [69]

$$
\begin{aligned}
\frac{\partial \rho}{\partial t}= & -i\left[H_{\mathrm{eff}}, \rho\right]+\gamma_{c} L[a] \rho \\
& +\gamma_{m}\left(n_{\mathrm{th}}+1\right) L[b] \rho+\gamma_{m} n_{\mathrm{th}} L\left[b^{\dagger}\right] \rho
\end{aligned}
$$

where $L[o] \rho=o \rho o^{\dagger}-\left(o^{\dagger} o \rho+\rho o^{\dagger} o\right) / 2$ denotes a Lindbland term for an operator $o$; $n_{\mathrm{th}}$ is the mean number of the thermal phonon, given by the Bose-Einstein statistics $n_{\text {th }}=$ $\left[\exp \left(\hbar \omega_{m} / k_{B} T\right)-1\right]^{-1}$ with the Boltzmann constant $k_{B}$ and the environmental temperature $T$. The temperature effect on the photon is neglected, because we assume that the optical frequency is much higher than the mechanical one. The
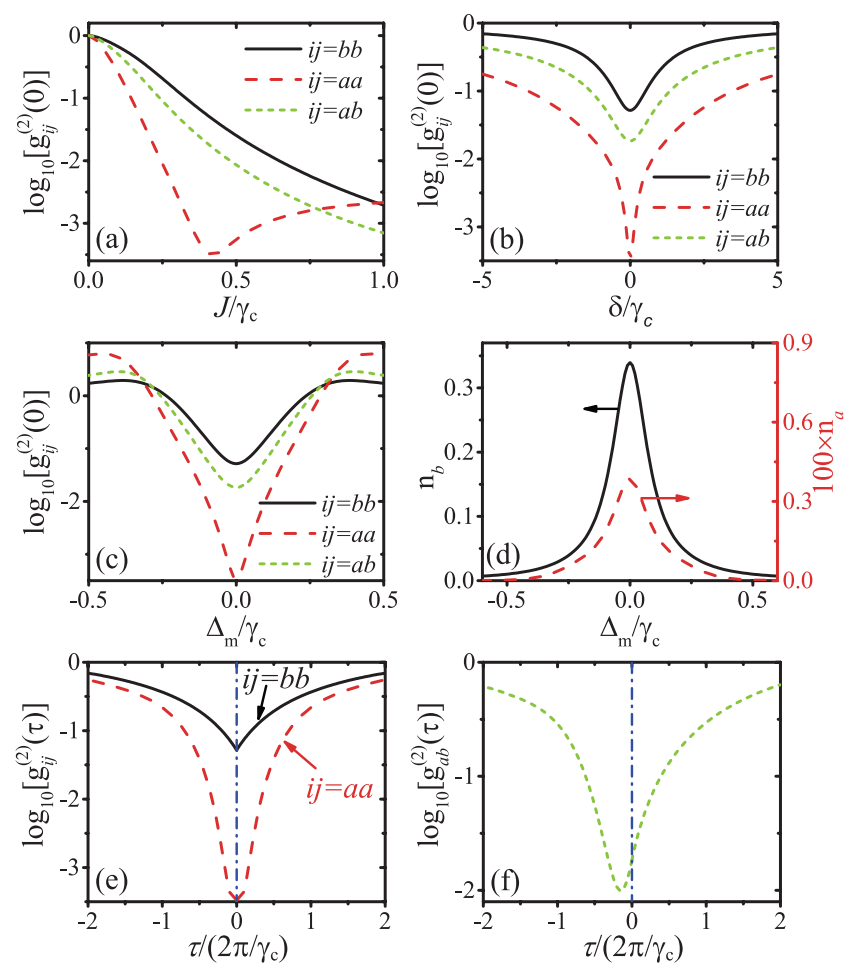

FIG. 2: (Color online) $\log _{10} g_{i j}^{(2)}(0)(i j=b b, a a, a b)$ are plotted (a) as functions of the effective coupling strength $J / \gamma_{c}$; (b) as functions of the detuning $\delta / \gamma_{c} \equiv\left(\omega_{c}-\omega_{L}-2 \omega_{d}\right) / \gamma_{c}$; (c) as functions of the detuning $\Delta_{m} / \gamma_{c}$. (d) Mean phonon number $n_{b}$ and photon number $100 \times n_{a}$ are plotted as functions of the detuning $\Delta_{m} / \gamma_{c}$. $\log _{10} g_{i j}^{(2)}(\tau)$ is plotted as a function of the normalized time delay $\tau /\left(2 \pi / \gamma_{c}\right)$ in (e) and (f). $\Delta=\Delta_{m}=0$ in (a); $\omega_{0}=\omega_{d}$ and $J=0.406 \gamma_{c}$ in (b); $J=0.406 \gamma_{c}$ and $\Delta=2 \Delta_{m}$ in (c) and (d); $\Delta=\Delta_{m}=0$ and $J=0.406 \gamma_{c}$ in (e) and (f). The other parameters are $\varepsilon=0.05 \gamma_{c}, \gamma_{m}=\gamma_{c} / 10$, and $n_{\mathrm{th}}=10^{-4}$.

second-order correlation functions can be calculated by solving the master equation in Eq. (12) numerically within a truncated Fock space.

\section{ANTICORRELATED PHONONS AND PHOTONS}

Figure 2(a) displays $\log _{10} g_{i j}^{(2)}(0)(i j=b b, a a, a b)$ as functions of the effective coupling strength $J / \gamma_{c}$ for $\Delta=\Delta_{m}=$ 0. $\log _{10} g_{b b}^{(2)}(0)$ and $\log _{10} g_{a b}^{(2)}(0)$ decrease gradually with the increase of the effective coupling strength $J$. Similar phenomena were mentioned in doubly resonant nanocavities with second-order nonlinearity [70], where a strongly anticorrelation between the first- and second-harmonic photons was reported. Here, we propose to realize strongly anticorrelation between photons and phonons with quadratically optomechanical coupling, which may have important applications in building hybrid systems. Moreover, different from the monotone increases of $\log _{10} g_{b b}^{(2)}(0)$ and $\log _{10} g_{a b}^{(2)}(0)$, the minimum of $\log _{10} g_{a a}^{(2)}(0)$ appears with the effective coupling strength $J \approx 0.406 \gamma_{c}$. 

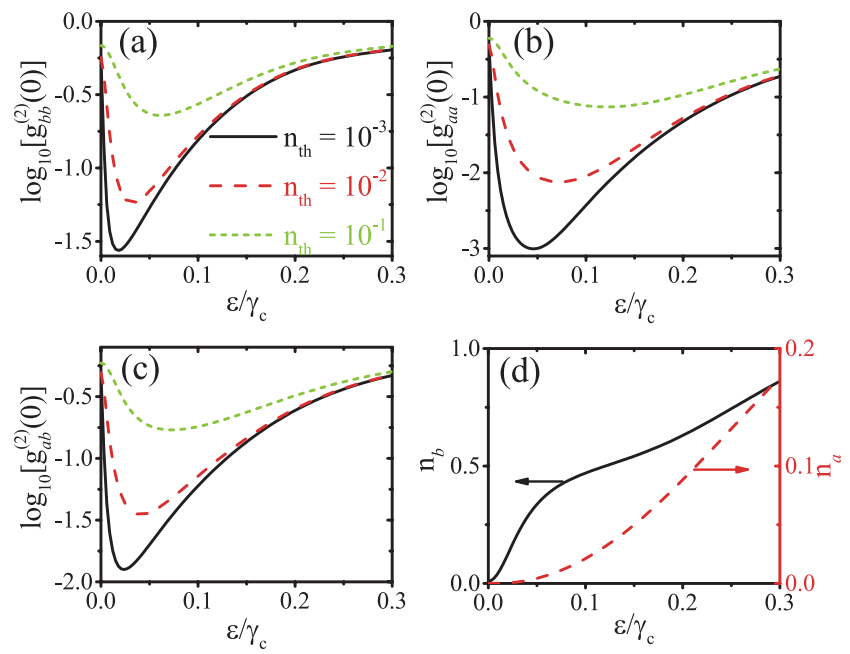

FIG. 3: (Color online) $\log _{10} g_{i j}^{(2)}(0)[$ (a) $i j=b b$, (b) $i j=a a$, (c) $i j=a b]$ is plotted as a function of the driving strength $\varepsilon / \gamma_{c}$ for different mean thermal phonon number $n_{\text {th }}$ [solid curve for $n_{\mathrm{th}}=10^{-3}$; dashed curve for $n_{\mathrm{th}}=10^{-2}$; dotted curve for $n_{\mathrm{th}}=10^{-1} \mathrm{]}$. (d) Mean phonon number $n_{b}$ and photon number $n_{a}$ are plotted as functions of the driving strength $\varepsilon / \gamma_{c}$ for mean thermal phonon number $n_{\mathrm{th}}=10^{-2}$. The other parameters are $\Delta=\Delta_{m}=0, J=0.406 \gamma_{c}$, and $\gamma_{m}=\gamma_{c} / 10$.

The monotone increases of $\log _{10} g_{b b}^{(2)}(0)$ and $\log _{10} g_{a b}^{(2)}(0)$ can be understood by the energy spectrum shown in Fig. 1. When the mechanical mode is driven by field with the frequency $\omega_{d}=\omega_{0}$, we can realize the phonon blockade in analogy to the cavity QED [7-14]: the transition from $\left|0_{0}\right\rangle$ to $\left|1_{0}\right\rangle$ is enhanced with resonant phonon absorption, while the transition from $\left|1_{0}\right\rangle$ to $\left|2_{ \pm 1}\right\rangle$ is blocked for detuning $\sqrt{2} J$. The anti-correlation between the photons and phonons for $g_{a b}^{(2)}(0)<1$ can be understood in a similar way: the the transition from $\left|2_{ \pm 1}\right\rangle$ to $\left|3_{ \pm 1}\right\rangle$ is also blocked for detuning $(\sqrt{6}-\sqrt{2}) J$. However, the appearing of the minimum of $\log _{10} g_{a a}^{(2)}(0)$ with the effective coupling strength $J \approx$ $0.406 \gamma_{c}$ cannot be explained by the same way with nonlinear energy spectrum of the system. This abnormal phenomenon results from the destructive interference between different paths for two-photon excitation in the optical mode [71] and we will give a detailed explanation in the next section.

There are two external driving fields applied to the system simultaneously: a strong optical field with frequency $\omega_{L}$ and a weak mechanical driving field with frequency $\omega_{d}$. $\log _{10} g_{i j}^{(2)}(0)(i j=b b, a a, a b)$ are plotted as functions of the detuning $\delta / \gamma_{c} \equiv\left(\omega_{c}-\omega_{L}-2 \omega_{d}\right) / \gamma_{c}$ in Fig. 2(b) and detuning $\Delta_{m}=\Delta / 2$ in Fig. 2(c). In Fig. 2(b), we set $\omega_{0}=\omega_{d}$ and the detunings $\delta$ is changed by tuning the frequency $\omega_{L}$; in Fig. 2(c), we set $\omega_{L}=\omega_{c}-2 \omega_{0}$ and the detuning $\Delta_{m}$ $(\Delta)$ is changed by tuning the frequency $\omega_{d}$. The figures show that the photon blockade, phonon blockade, and strongly anticorrelated photons and phonons are much more rigid against the tuning of frequency $\omega_{L}$ but more sensitive to the tuning of frequency $\omega_{d}$.

Mean phonon number $n_{b}$ and photon number $n_{a}$ for pho-
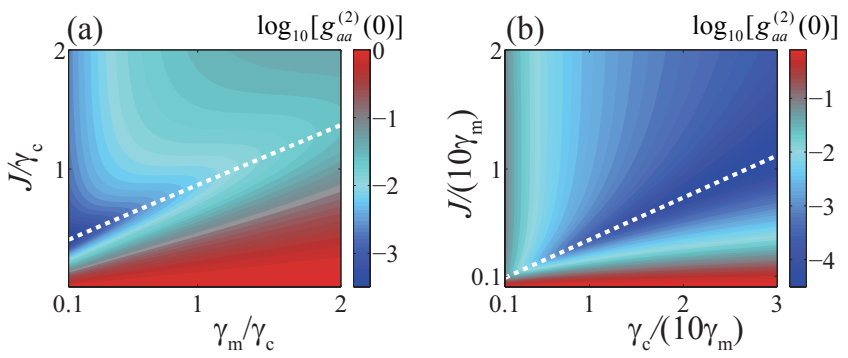

FIG. 4: (Color online) (a) Contour plot of $\log _{10} g_{a a}^{(2)}(0)$ as a function of the damping rate $\gamma_{m} / \gamma_{c}$ and the effective coupling strength $J / \gamma_{c}$ for $\varepsilon=0.05 \gamma_{c}$; (a) contour plot of $\log _{10} g_{a a}^{(2)}(0)$ vs the damping rate $\gamma_{c} /\left(10 \gamma_{m}\right)$ and the effective coupling strength $J /\left(10 \gamma_{m}\right)$ for $\varepsilon=0.5 \gamma_{m}$. The white dashed line refers to Eq. (22). The other parameters are $\Delta=\Delta_{m}=0$ and $n_{\mathrm{th}}=10^{-4}$.

ton blockade, phonon blockade and strongly anticorrelated photons and phonons are plotted as functions of the detuning $\Delta / \gamma_{c}$ in Fig. 2(d). As the generation of a single photon needs annihilating two single phonons, the efficient for singlephoton generation is much lower than the one for singlephonon generation. The second-order correlation function $g_{i j}^{(2)}(\tau)(i j=b b, a a, a b)$ is plotted as a function of the normalized time delay $\tau /\left(2 \pi / \gamma_{c}\right)$ in Fig. 2 (e) and (f) for $\Delta=\Delta_{m}=0$ and $J=0.406 \gamma_{c}$. The time duration for photon blockade, phonon blockade and strongly anticorrelated photons and phonons is of the order of the lifetime of the photons in the cavity. The cross-correlation function for anticorrelated photons and phonons is asymmetric for $\tau>0$ and $\tau<0$.

$\log _{10} g_{i j}^{(2)}(0)$ is plotted as a function of the mechanical driving strength $\varepsilon / \gamma_{c}$ for different mean thermal phonon numbers $n_{\text {th }}$ in Fig. 3: (a) $i j=b b$, (b) $i j=a a$, (c) $i j=a b$. Mean phonon number $n_{b}$ and photon number $n_{a}$ are plotted as functions of the mechanical driving strength $\varepsilon$ in Fig. 3(d). Clearly, the thermal phonons have a detrimental effect on the realization of photon blockade, phonon blockade and strongly anticorrelated photons and phonons. A proper increase of the mechanical driving strength $\varepsilon$ can increase the number of mean phonons and photons and this is also helpful to overcome the detrimental effect induced by the thermal phonons. But if the mechanical driving strength becomes too strong, the phonons and photons tend to behave classically.

\section{UNCONVENTIONAL PHOTON BLOCKADE}

To understand the origin of the strong photon antibunching appearing with weak coupling strength $J \approx 0.406 \gamma_{c}$, we now examine the paths for two-photon excitation. As illustrated in Fig. 1 (left), there are two transition paths for two-photon generation: $|0,4\rangle \rightarrow|1,2\rangle \rightarrow|2,0\rangle$ and $|1,1\rangle \rightarrow|1,2\rangle \rightarrow|2,0\rangle$. The strong photon antibunching can be explained using the destructive interference between the two different paths of two-photon generation. The occupation probabilities in states $|1,2\rangle$ and $|2,0\rangle$ become zero when the transition matrix elements of these two paths of photon excitation have the same 
amplitude but different phase. To examine this explanation, following the method given in Ref. [71], we will derive the optimal conditions for strong photon antibunching with the resonant driving condition $\Delta=\Delta_{m}=0$.

As the mechanical driving field is not so strong that the average photon and phonon numbers $n_{a}$ and $n_{b}$ are small, i.e. $n_{a} \ll 1$ and $n_{b} \ll 1$, we can expand the wave function $|\psi\rangle$ in the Fock-state basis $|n, m\rangle$ truncated to the two-photon and four-phonon states. That is, we assume

$$
\begin{aligned}
|\psi\rangle= & C_{00}|0,0\rangle+C_{01}|0,1\rangle \\
& +C_{02}|0,2\rangle+C_{10}|1,0\rangle \\
& +C_{03}|0,3\rangle+C_{11}|1,1\rangle \\
& +C_{04}|0,4\rangle+C_{12}|1,2\rangle+C_{20}|2,0\rangle
\end{aligned}
$$

where the coefficients satisfy $C_{00} \approx 1 \gg C_{01} \gg C_{02}, C_{10} \gg$ $C_{03}, C_{11} \gg C_{04}, C_{12}, C_{20}$. The coefficient $\left|C_{n m}\right|^{2}$ denotes the occupying probability in the state $|n, m\rangle$. Substituting the wave function in Eq. (13) and the effective Hamiltonian in Eq. (8) with $\Delta=\Delta_{m}=0$ into the Schrödinger's equation $i \partial_{t}|\psi\rangle=H_{\text {eff }}|\psi\rangle$, the dynamical equations for the coefficients $C_{n m}$ can be obtained by taking account of the dampings of the optical and mechanical modes. The steady-state values of the coefficients $C_{n m}$ are determined by the equations

$$
\begin{gathered}
0=-\frac{\gamma_{m}}{2} C_{01}-i \varepsilon C_{00}, \\
0=-\frac{\gamma_{c}}{2} C_{10}-i \sqrt{2} J C_{02}, \\
0=-\gamma_{m} C_{02}-i \sqrt{2} J C_{10}-i \sqrt{2} \varepsilon C_{01}, \\
0=-\frac{\gamma_{c}+\gamma_{m}}{2} C_{11}-i \sqrt{6} J C_{03}-i \varepsilon C_{10}, \\
0=-\frac{3 \gamma_{m}}{2} C_{03}-i \sqrt{6} J C_{11}-i \sqrt{3} \varepsilon C_{02}, \\
0=-\gamma_{c} C_{20}-i 2 J C_{12}, \\
0=-\frac{\gamma_{c}+2 \gamma_{m}}{2} C_{12}-i 2 \sqrt{3} J C_{04}-i 2 J C_{20} \\
\quad-i \sqrt{2} \varepsilon C_{11}, \\
0=-2 \gamma_{m} C_{04}-i 2 \sqrt{3} J C_{12}-i 2 \varepsilon C_{03} .
\end{gathered}
$$

To derive the optimal condition for photon blockade [i.e., $g_{a a}^{(2)}(0) \approx 0$ ], we set $C_{20}=0$, then the optimal effective coupling strength is obtained as

$$
J_{\mathrm{opt}}=\sqrt{\frac{1}{8}\left(2 \gamma_{m}+\gamma_{c}\right)\left(\gamma_{m}+\gamma_{c}\right)} .
$$

Substituting $\gamma_{m}=\gamma_{c} / 10$ into the above equation, we have $J_{\text {opt }} \approx 0.406 \gamma_{c}$, which is consistent well with the numerical results shown in Fig. 2(b).

Figure 4 shows the contour plot of $\log _{10} g_{a a}^{(2)}(0)$ as a function of the damping rate $\gamma_{m} / \gamma_{c}$ [or $\gamma_{c} /\left(10 \gamma_{m}\right)$ ] and the effective coupling strength $J / \gamma_{c}$. The white dashed line in Fig. 4 is the optimal effective coupling strength, given by Eq. (22). It is clear that the optimal effective coupling strength, given by Eq. (22), agrees well with the numerical results. This suggests that the strong photon blockade appearing with weak coupling strength is induced by the destructive interference between two different paths of two-photon excitation. We can call the interference-based photon blockade as unconventional photon blockade, which is similar to the unconventional photon blockade in a weakly nonlinear system of photonic molecule [71-81].

It is worth mentioning that our study is different from that of nonlinear photonic molecules. The main difference is that there are two separate energy scales in the weakly nonlinear photonic molecules [49,71-81], one is large linear coupling strength between coupled cavity modes, and the second is small nonlinearity (up to hundred times smaller than the photon damping rate). Here, in the quadratically coupled optomechanical systems, there is only one parameter, i.e. the effective (nonlinear) coupling strength $J$, which is the order of the damping rate of the optical mode [see Eq. (22)].

\section{CORRELATED PHONONS AND PHOTONS}

Different from the previous two sections, here, we assume that the system works in the strong coupling condition, i.e., $J=5 \gamma_{c} . \quad \log _{10} g_{i j}^{(2)}(0)(i j=b b, a a, a b)$ are plotted as functions of the detuning $\Delta_{m}$ in Fig. 5(a), where $\omega_{L}=\omega_{c}-2 \omega_{0}$ and $\Delta=2 \Delta_{m}$ as shown in Fig. 2(c). Figures 5(b) and 5(c) are the local enlarged drawings of Fig. 5(a). As shown in Fig. 5(b), there are peaks and dips around the points $\Delta_{m} / \gamma_{c}=5 / \sqrt{2}, 5 \sqrt{6} / 3$, and 5 [blue dashed-dotted lines in Fig. 5(b)], which are corresponding to the resonant transitions $\left|0_{0}\right\rangle \rightarrow\left|2_{ \pm 1}\right\rangle,\left|0_{0}\right\rangle \rightarrow\left|3_{ \pm 1}\right\rangle$, and $\left|0_{0}\right\rangle \rightarrow\left|4_{ \pm 1}\right\rangle$ with energy levels shown in Fig. 1.

There is another interesting phenomenon that all the second-order correlation functions become larger (even much larger) than 1, i.e., $\log _{10} g_{i j}^{(2)}(0)>0(i j=b b, a a, a b)$, in the areas between the peaks and dips, such as the area shown in Fig. 5(c). These imply that the photon pairs and phonon pairs can be generated simultaneously and the generated photons and phonons are correlated with each other. Future applications could include the two-photon gateway, two-phonon gateway, and the correlated photon-phonon gateway [82].

$\log _{10} g_{i j}^{(2)}(\tau)(i j=b b, a a, a b)$ are plotted as a function of the normalized time delay $\tau /\left(2 \pi / \gamma_{c}\right)$ in Figs. 5(d), 5(e) and 5(f) for the detuning $\Delta_{m}=3.9 \gamma_{c} \cdot \log _{10} g_{i j}^{(2)}(\tau)$ shows an oscillation behavior with the periods $2 \pi /\left(n \Delta_{m}\right)$ ( $n$ is a positive integer). These oscillation behaviors with the periods $2 \pi /\left(n \Delta_{m}\right)$ come from the population oscillation between the states $|n, m\rangle \rightarrow|n, m+1\rangle$ with detuning $\Delta_{m}=3.9 \gamma_{c}$ in the weak driving condition $\varepsilon \ll \gamma_{c}$. The time durations for the generations of phonon (photon) pairs and correlated photons and phonons are of the order of the lifetime of the phonons. The cross-correlation function for correlated photons and phonons is asymmetric for $\tau>0$ and $\tau<0$. 

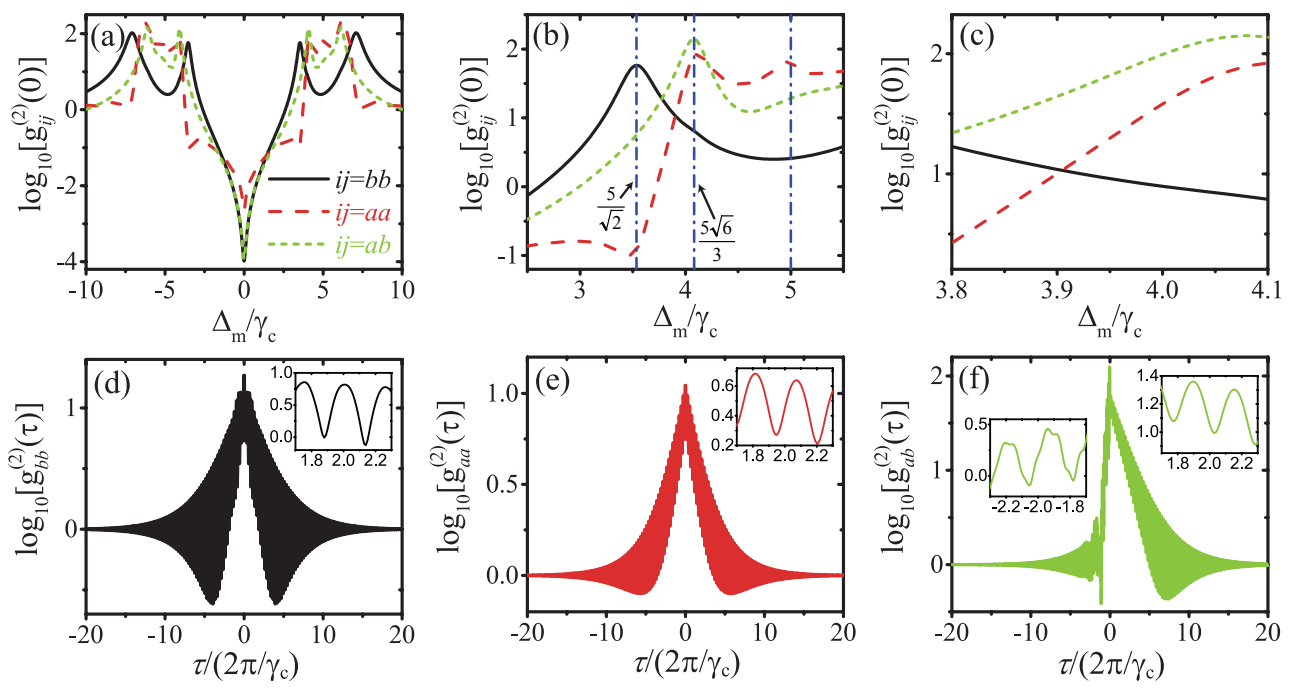

FIG. 5: (Color online) (a) $\log _{10} g_{i j}^{(2)}(0)(i j=b b, a a, a b)$ is plotted as a function of the detuning $\Delta_{m} / \gamma_{c}$; (b) and (c) are the local enlarged drawings of (a). The blue dashed-dotted lines in (b) refer to $\Delta_{m} / \gamma_{c}=5 / \sqrt{2}, 5 \sqrt{6} / 3$ and $5 \log _{10} g_{i j}^{(2)}(\tau)[(\mathrm{d}) i j=b b$, (e) $i j=a a$, (f) $i j=a b]$ is plotted as a function of the normalized time delay $\tau /\left(2 \pi / \gamma_{c}\right)$ in (d)-(f) for $\Delta_{m}=3.9 \gamma_{c}$. The other parameters are $\varepsilon=0.05 \gamma_{c}$, $J=5 \gamma_{c}, \omega_{L}=\omega_{c}-2 \omega_{0}, \Delta=2 \Delta_{m}, \gamma_{m}=\gamma_{c} / 10$, and $n_{\mathrm{th}}=10^{-4}$.

$\log _{10} g_{i j}^{(2)}(0)$ is plotted as a function of the mechanical driving strength $\varepsilon / \gamma_{c}$ for different mean thermal phonon numbers $n_{\text {th }}$ in Fig. 6: (a) $i j=b b$, (b) $i j=a a$, (c) $i j=a b$. Mean phonon number $n_{b}$ and photon number $n_{a}$ are plotted as functions of the mechanical driving strength $\varepsilon$ in Fig. 6(d). Similarly to the case in Fig. 3, the thermal phonons have a detrimental effect on the realization of bunching phonons (phonons) and correlated photons and phonons. A proper increase of the mechanical driving strength $\varepsilon$ can increase the number of mean phonons and photons and this is also helpful to overcome the detrimental effect induced by the thermal phonons. But if the mechanical driving strength becomes too strong, the phonons and photons tend to behave classically.

\section{DISCUSSIONS AND CONCLUSIONS}

The first and also the most important condition required to observe anticorrelated and correlated photons and phonons in quadratical coupled optomechanical systems is the well resolved sideband limit, i.e., $\omega_{m} \gg \gamma_{c}$. This requirement could be reached for the optomechanical crystals as indicated in Ref. [60] by numerical simulations, where the simulated parameters are: mechanical resonance frequency $\omega_{m} / 2 \pi=225$ $\mathrm{MHz}$ and optical damping rate $\gamma_{c} / 2 \pi=20 \mathrm{MHz}$. Another candidate system is the Fabry-Perot cavity with membranein-the-middle [61-64] and the resolved sideband limit was reached in Ref. [63] with mechanical resonance frequency $\omega_{m} / 2 \pi \approx 788 \mathrm{kHz}$ and optical damping rate $\gamma_{c} / 2 \pi=177$ $\mathrm{kHz}$. In addition, the quadratical couplings have been explored in a number of other optomechanical systems [6568]. Secondly, it is a outstanding challenge to detect single phonons directly in the experiments. The measurements of
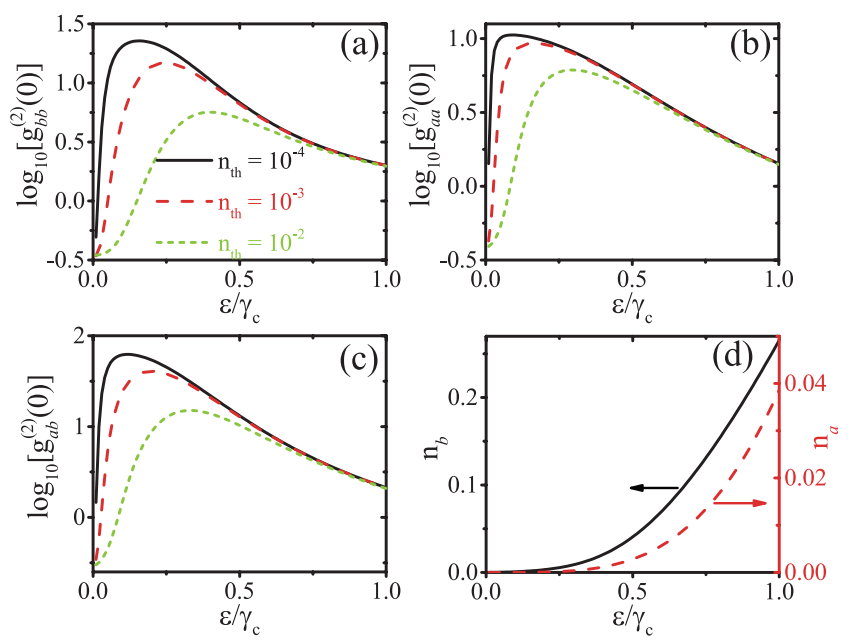

FIG. 6: (Color online) $\log _{10} g_{i j}^{(2)}(0)[(a) i j=b b$, (b) $i j=a a$, (c) $i j=a b]$ is plotted as a function of the driving strength $\varepsilon / \gamma_{c}$ for different mean thermal phonon number $n_{\text {th }}$ [solid curve for $n_{\text {th }}=$ $10^{-4}$; dashed curve for $n_{\mathrm{th}}=10^{-3}$; dotted curve for $\left.n_{\mathrm{th}}=10^{-2}\right]$. (d) Mean phonon number $n_{b}$ (solid curve) and photon number $n_{a}$ (dashed curve) are plotted as functions of the driving strength $\varepsilon / \gamma_{c}$ for mean thermal phonon number $n_{\mathrm{th}}=10^{-4}$. The other parameters are $\Delta_{m}=3.9 \gamma_{c}, \Delta=2 \Delta_{m}, J=5 \gamma_{c}$, and $\gamma_{m}=\gamma_{c} / 10$.

the correlation of the phonons and the cross-correlation between photons and phonons can be realized by converting the mechanical signals into optical signals through auxiliary optomechanical couplings [23-25], which have been realized in a recent experiment [24].

In summary, we have studied the photon, phonon statistics and the cross-correlation between photons and phonons 
in a quadratically coupled optomechanical system. We show that photon blockade, phonon blockade, and strong anticorrelation between photons and phonons can be observed in the same parameter area. Phonon blockade and strong anticorrelation between photons and phonons can be understood by the nonlinear energy spectrum of the system, while the photon blockade with weak nonlinear coupling strength can only be explanted by the destructive interference between different paths for two-photon excitation. The combination of photon blockade, phonon blockade, and strongly anticorrelated photons and phonons provides us a way to generate anticorrelated single photons and single phonons. Further more, in the strongly nonlinear coupling condition, photon pairs and phonon pairs can be generated simultaneously, and the photon and phonon pairs are correlated with each other, which can be used to generate two-photon gateway, two-phonon gateway, and the correlated photon-phonon gateway [82].

\section{Acknowledgement}

X.W.X. is supported by the National Natural Science Foundation of China (NSFC) under Grants No.11604096 and the Startup Foundation for Doctors of East China Jiaotong University under Grant No. 26541059. A.X.C. is supported by NSFC under Grant No. 11775190. Y.X.L. is supported by the National Basic Research Program of China(973 Program) under Grant No. 2014CB921401, the Tsinghua University Initiative Scientific Research Program, and the Tsinghua National Laboratory for Information Science and Technology (TNList) Cross-discipline Foundation.
[1] V. Scarani, H. Bechmann-Pasquinucci, N. J. Cerf, M. Dusek, N. Lutkenhaus, and M. Peev, The security of practical quantum key distribution, Rev. Mod. Phys. 81, 1301 (2009).

[2] J. W. Pan, Z. B. Chen, C. Y. Lu, H. Weinfurter, A. Zeilinger, and M. Zukowski, Multiphoton entanglement and interferometry, Rev. Mod. Phys. 84, 777 (2012).

[3] B. Lounis and M. Orrit, Single-photon sources, Rep. Prog. Phys. 68, 1129 (2005).

[4] S. Buckley, K. Rivoire, and J. Vučković, Engineered quantum dot single-photon sources, Rep. Prog. Phys. 75, 126503 (2012)

[5] G. C. Shan, Z. Q. Yin, C. H. Shek, and W. Huang, Single photon sources with single semiconductor quantum dots, Front. Phys. 9(2), 170 (2014).

[6] A. Imamoğlu, H. Schmidt, G. Woods, and M. Deutsch, Strongly Interacting Photons in a Nonlinear Cavity, Phys. Rev. Lett. 79, 1467 (1997).

[7] K. M. Birnbaum, A. Boca, R. Miller, A. D. Boozer, T. E. Northup, and H. J. Kimble, Photon blockade in an optical cavity with one trapped atom, Nature (London) 436, 87 (2005).

[8] B. Dayan, A. S. Parkins, T. Aoki, E. P. Ostby, K. J. Vahala, and H. J. Kimble, A Photon Turnstile Dynamically Regulated by One Atom, Science 319, 1062 (2008).

[9] F. Dubin, C. Russo, H. G. Barros, A. Stute, C. Becher, P. O. Schmidt, and R. Blatt, Quantum to Classical Transition in a Single-Ion Laser, Nature Phys. 6, 350 (2010).

[10] A. Faraon, I. Fushman, D. Englund, N. Stoltz, P. Petro, and J. Vuckovic, Coherent generation of non-classical light on a chip via photon-induced tunnelling and blockade, Nat. Phys. 4, 859 (2008).

[11] X. Ding, Y. He, Z.-C. Duan, N. Gregersen, M.-C. Chen, S. Unsleber, S. Maier, C. Schneider, M. Kamp, S. Höfling, C. Y. $\mathrm{Lu}$, and J. W. Pan, On-Demand Single Photons with High Extraction Efficiency and Near-Unity Indistinguishability from a Resonantly Driven Quantum Dot in a Micropillar, Phys. Rev. Lett. 116, 020401 (2016).

[12] C. Lang, D. Bozyigit, C. Eichler, L. Steffen, J. M. Fink, A. A. Abdumalikov, Jr., M. Baur, S. Filipp, M. P. da Silva, A. Blais, and A. Wallraff, Observation of Resonant Photon Blockade at Microwave Frequencies Using Correlation Function Measurements, Phys. Rev. Lett. 106, 243601 (2011).

[13] A. J. Hoffman, S. J. Srinivasan, S. Schmidt, L. Spietz, J. Aumentado, H. E. Türeci, and A. A. Houck, Dispersive Photon
Blockade in a Superconducting Circuit, Phys. Rev. Lett. 107, 053602 (2011).

[14] Y. X. Liu, X. W. Xu, A. Miranowicz, and F. Nori, From blockade to transparency: Controllable photon transmission through a circuit-QED system, Phys. Rev. A 89, 043818 (2014).

[15] K. C. Schwab and M. L. Roukes, Putting mechanics into quantum mechanics, Phys. Today 58(7), 36 (2005).

[16] M. Poot and H. S. J. van der Zant, Mechanical systems in the quantum regime, Phys. Rep. 511, 273 (2012).

[17] K. Vahala, M. Herrmann, S. Knünz, V. Batteiger, G. Saathoff, T. W. Hänsch, and Th. Udem, A phonon laser, Nature Phys. 5, 682 (2009).

[18] I. S. Grudinin, H. Lee, O. Painter, and K. J. Vahala, Phonon Laser Action in a Tunable Two-Level System, Phys. Rev. Lett. 104, 083901 (2010).

[19] H. Wang, Z. Wang, J. Zhang, Ç. K. Özdemir, L. Yang, and Y. $\mathrm{X}$. Liu, Phonon amplification in two coupled cavities containing one mechanical resonator, Phys. Rev. A 90, 053814 (2014).

[20] Y. X. Liu, A. Miranowicz, Y. B. Gao, J. Bajer, C. P. Sun, and F. Nori, Qubit-induced phonon blockade as a signature of quantum behavior in nanomechanical resonators, Phys. Rev. A 82, 032101 (2010).

[21] Sh. Barzanjeh and D. Vitali, Phonon Josephson junction with nanomechanical resonators, Phys. Rev. A 93, 033846 (2016).

[22] N. Didier, S. Pugnetti, Y. M. Blanter, and R. Fazio, Detecting phonon blockade with photons, Phys. Rev. B 84, 054503 (2011).

[23] T. Ramos, V. Sudhir, K. Stannigel, P. Zoller, and T. J. Kippenberg, Nonlinear Quantum Optomechanics via Individual Intrinsic Two-Level Defects, Phys. Rev. Lett. 110, 193602 (2013).

[24] J. D. Cohen, S. M. Meenehan, G. S. MacCabe, S. Groblacher, A. H. Safavi-Naeini, F. Marsili, M. D. Shaw, and O. Painter, Phonon counting and intensity interferometry of a nanomechanical resonator, Nature (London) 520, 522 (2015).

[25] X. W. Xu, A. X. Chen, and Y. X. Liu, Phonon blockade in a nanomechanical resonator resonantly coupled to a qubit, Phys. Rev. A 94, 063853 (2016).

[26] A. Miranowicz, J. Bajer, N. Lambert, Y. X. Liu, and F. Nori, Tunable multiphonon blockade in coupled nanomechanical resonators, Phys. Rev. A 93, 013808 (2016).

[27] X. Wang, A. Miranowicz, H. R. Li, and F. Nori, Method for observing robust and tunable phonon blockade in a nanome- 
chanical resonator coupled to a charge qubit, Phys. Rev. A 93, 063861 (2016).

[28] X. W. Xu, H. Wang, J. Zhang, and Y. X. Liu, Engineering of nonclassical motional states in optomechanical systems, Phys. Rev. A 88, 063819 (2013).

[29] M. Wallquist, K. Hammerer, P. Rabl, M. Lukin, and P. Zoller, Hybrid quantum devices and quantum engineering, Phys. Scr. T137, 014001 (2009).

[30] Z. L. Xiang, S. Ashhab, J. Q. You, and F. Nori, Hybrid quantum circuits: Superconducting circuits interacting with other quantum systems, Rev. Mod. Phys. 85, 623 (2013).

[31] X. Gu, A. F. Kockum, A. Miranowicz, Y. X. Liu, and F. Nori, Microwave photonics with superconducting quantum circuits, Phys. Rep. 718-719, 1 (2017).

[32] O. Arcizet, V. Jacques, A. Siria, P. Poncharal, P. Vincent, and S. Seidelin, A single nitrogen-vacancy defect coupled to a nanomechanical oscillator, Nat. Phys. 7, 879 (2011).

[33] E. R. MacQuarrie, T. A. Gosavi, N. R. Jungwirth, S. A. Bhave, and G. D. Fuchs, Mechanical Spin Control of Nitrogen-Vacancy Centers in Diamond, Phys. Rev. Lett. 111, 227602 (2013).

[34] C. K. Law, Interaction between a moving mirror and radiation pressure: A Hamiltonian formulation, Phys. Rev. A 51, 2537 (1995).

[35] T. J. Kippenberg and K. J. Vahala, Cavity Optomechanics: Back-Action at the Mesoscale, Science 321, 1172 (2008).

[36] F. Marquardt and S. M. Girvin, Optomechanics, Physics 2, 40 (2009).

[37] M. Aspelmeyer, P. Meystre, and K. Schwab, Quantum optomechanics, Phys. Today 65(7), 29 (2012).

[38] M. Aspelmeyer, T. J. Kippenberg, and F. Marquardt, Cavity Optomechanics, Rev. Mod. Phys. 86, 1391 (2014).

[39] M. Metcalfe, Applications of cavity optomechanics, Appl. Phys. Rev. 1, 031105 (2014).

[40] Y. L. Liu, C. Wang, J. Zhang, and Y. X. Liu, Cavity optomechanics: Manipulating photons and phonons towards the singlephoton strong coupling, Chin. Phys. B 27, 024204 (2018).

[41] P. Rabl, Photon Blockade Effect in Optomechanical Systems, Phys. Rev. Lett. 107, 063601 (2011).

[42] A. Nunnenkamp, K. Børkje, and S. M. Girvin, Single-Photon Optomechanics, Phys. Rev. Lett. 107, 063602 (2011).

[43] A. Kronwald, M. Ludwig, and F. Marquardt, Full photon statistics of a light beam transmitted through an optomechanical system, Phys. Rev. A 87, 013847 (2013).

[44] X. W. Xu, Y. J. Li, and Y. X. Liu, Photon-induced tunneling in optomechanical systems, Phys. Rev. A 87, 025803 (2013).

[45] J. Q. Liao and F. Nori, Photon blockade in quadratically coupled optomechanical systems, Phys. Rev. A 88, 023853 (2013).

[46] J. Q. Liao and C. K. Law, Correlated two-photon scattering in cavity optomechanics, Phys. Rev. A 87, 043809 (2013).

[47] D. Hu, S. Y. Huang, J. Q. Liao, L. Tian, and H. S. Goan, Quantum coherence in ultrastrong optomechanics, Phys. Rev. A 91, 013812(2015).

[48] K. Stannigel, P. Komar, S. J. M. Habraken, S. D. Bennett, M. D. Lukin, P. Zoller, and P. Rabl, Optomechanical Quantum Information Processing with Photons and Phonons, Phys. Rev. Lett. 109, 013603 (2012).

[49] P. Kómár, S. D. Bennett, K. Stannigel, S. J. M. Habraken, P. Rabl, P. Zoller, and M. D. Lukin, Single-photon nonlinearities in two-mode optomechanics, Phys. Rev. A 87, 013839 (2013).

[50] X. W. Xu and Y. J. Li, Antibunching photons in a cavity coupled to an optomechanical system, J. Phys. B: At. Mol. Opt. Phys. 46, 035502 (2013).

[51] V. Savona, Unconventional photon blockade in coupled optomechanical systems, arXiv:1302.5937 [quant-ph].
[52] X. Y. Lü, Y. Wu, J. R. Johansson, H. Jing, J. Zhang, and F. Nori, Squeezed Optomechanics with Phase-Matched Amplification and Dissipation, Phys. Rev. Lett. 114, 093602 (2015).

[53] T. S. Yin, X. Y. Lü, L. L. Zheng, M. Wang, S. Li, and Y. Wu, Nonlinear effects in modulated quantum optomechanics, Phys. Rev. A 95, 053861 (2017).

[54] H. Xie, G. W. Lin, X. Chen, Z. H. Chen, and X. M. Lin, Singlephoton nonlinearities in a strongly driven optomechanical system with quadratic coupling, Phys. Rev. A 93, 063860 (2016).

[55] H. Seok and E. M. Wright, Antibunching in an optomechanical oscillator, Phys. Rev. A 95, 053844 (2017).

[56] H. Xie, C. G. Liao, X. Shang, M. Y. Ye, and X. M. Lin, Phonon blockade in a quadratically coupled optomechanical system, Phys. Rev. A 96, 013861 (2017)

[57] H. Q. Shi, X. T. Zhou, X. W. Xu, and N. H. Liu, Tunable phonon blockade in quadratically coupled optomechanical systems, Sci. Rep. 8, 2212 (2018).

[58] S. Carlig and M. A. Macovei, Quantum correlations among optical and vibrational quanta, Phys. Rev. A 89, 053803 (2014).

[59] L. G. Si, H. Xiong, M. S. Zubairy, and Y. Wu, Optomechanically induced opacity and amplification in a quadratically coupled optomechanical system, Phys. Rev. A 95, 033803 (2017)

[60] T. K. Paraïso, M. Kalaee, L. Zang, H. Pfeifer, F. Marquardt, and O. Painter, Position-Squared Coupling in a Tunable Photonic Crystal Optomechanical Cavity, Phys. Rev. X 5, 041024 (2015).

[61] N. E. Flowers-Jacobs, S.W. Hoch, J. C. Sankey, A. Kashkanova, A. M. Jayich, C. Deutsch, J. Reichel, and J. G. E. Harris, FiberCavity-Based Optomechanical Device, Appl. Phys. Lett. 101, 221109 (2012).

[62] J. D. Thompson, B. M. Zwickl, A. M. Jayich, F. Marquardt, S. M. Girvin, and J. G. E. Harris, Strong dispersive coupling of a high-finesse cavity to a micromechanical membrane, Nature (London) 452, 72 (2008).

[63] H. Xu, D. Mason, L. Jiang, and J. G. E. Harris, Topological energy transfer in an optomechanical system with exceptional points, Nature (London) 537, 80 (2016).

[64] H. Xu, U. Kemiktarak, J. Fan, S. Ragole, J. Lawall, and J. M. Taylor, Observation of optomechanical buckling transitions, Nat. Commun. 8, 14481 (2017).

[65] T. P. Purdy, D. W. C. Brooks, T. Botter, N. Brahms, Z.-Y. Ma, and D. M. Stamper-Kurn, Tunable Cavity Optomechanics with Ultracold Atoms, Phys. Rev. Lett. 105, 133602 (2010).

[66] J. T. Hill, Nonlinear Optics and Wavelength Translation via Cavity-Optomechanics, Ph.D. thesis, California Institute of Technology, 2013.

[67] C. Doolin, B. D. Hauer, P. H. Kim, A. J. R. MacDonald, H. Ramp, and J. P. Davis, Nonlinear Optomechanics in the Stationary Regime, Phys. Rev. A 89, 053838 (2014).

[68] G. A. Brawley, M. R. Vanner, P. E. Larsen, S. Schmid, A. Boisen, and W. P. Bowen, Nonlinear optomechanical measurement of mechanical motion, Nat. Commun. 7, 10988 (2016).

[69] H. J. Carmichael, An Open Systems Approach to Quantum Optics, Lecture Notes in Physics Vol. 18 (Springer-Verlag, Berlin, 1993).

[70] A. Majumdar and D. Gerace, Single-photon blockade in doubly resonant nanocavities with second-order nonlinearity, Phys. Rev. B 87, 235319 (2013).

[71] M. Bamba, A. Imamoglu, I. Carusotto, and C. Ciuti, Origin of strong photon antibunching in weakly nonlinear photonic molecules, Phys. Rev. A 83, 021802(R) (2011).

[72] T. C. H. Liew and V. Savona, Single Photons from Coupled Quantum Modes, Phys. Rev. Lett. 104, 183601 (2010).

[73] M.-A. Lemonde, N. Didier, and A. A. Clerk, Antibunching and unconventional photon blockade with Gaussian squeezed states, 
Phys. Rev. A 90, 063824 (2014).

[74] A. Majumdar, M. Bajcsy, A. Rundquist, and J. Vuckovic, Loss-Enabled Sub-Poissonian Light Generation in a Bimodal Nanocavity, Phys. Rev. Lett. 108, 183601 (2012).

[75] D. Gerace and V. Savona, Unconventional photon blockade in doubly resonant microcavities with second-order nonlinearity, Phys. Rev. A 89, 031803(R) (2014).

[76] O. Kyriienko, I. A. Shelykh, and T. C. H. Liew, Tunable singlephoton emission from dipolaritons, Phys. Rev. A 90, 033807 (2014).

[77] X. W. Xu and Y. Li, Strong photon antibunching of symmetric and antisymmetric modes in weakly nonlinear photonic molecules, Phys. Rev. A 90, 033809 (2014).

[78] X. W. Xu and Y. Li, Tunable photon statistics in weakly nonlinear photonic molecules, Phys. Rev. A 90, 043822 (2014).
[79] O. Kyriienko and T. C. H. Liew, Triggered single-photon emitters based on stimulated parametric scattering in weakly nonlinear systems, Phys. Rev. A 90, 063805 (2014).

[80] H. Z. Shen, Y. H. Zhou, and X. X. Yi, Tunable photon blockade in coupled semiconductor cavities, Phys. Rev. A 91, 063808 (2015).

[81] Y. H. Zhou, H. Z. Shen, and X. X. Yi, Unconventional photon blockade with second-order nonlinearity, Phys. Rev. A 92 , 023838 (2015).

[82] A. Kubanek, A. Ourjoumtsev, I. Schuster, M. Koch, P. W. H. Pinkse, K. Murr, and G. Rempe, Two-Photon Gateway in OneAtom Cavity Quantum Electrodynamics, Phys. Rev. Lett. 101, 203602 (2008). 